\title{
The role of stem cells in tumor targeting and growth suppression of gliomas
}

\author{
This article was published in the following Dove Press journal: \\ Biologics:Targets and Therapy \\ 4 April 20I I \\ Number of times this article has been viewed
}

\author{
Hossein Eskandary' \\ Mohsen Basiri' \\ Seyed Noureddin \\ Nematollahi-Mahani ${ }^{2}$ \\ Sepideh Mehravaran ${ }^{3}$ \\ 'Neuroscience Research Center, \\ 2Department of Anatomy, Afzalipour \\ School of Medicine, Kerman \\ University of Medical Sciences, \\ ${ }^{3}$ Afzal Research Institute, Kerman, Iran
}

\begin{abstract}
Glioma remains the most challenging solid organ tumor to treat successfully. Based on the capacity of stem cells to migrate extensively and target invading glioma cells, the transplantation of stem cells as a cell-based delivery system may provide additional tools for the treatment of gliomas. In addition to the use of modified stem cells for the delivery of therapeutic agents, unmodified stem cells have been shown to have growth-suppressing effects on tumors in vitro and in vivo. This review outlines the probable factors involved in tumor tropism and tumor growth suppression, with a specific focus on the use of unmodified stem cells in the treatment of gliomas. Based on these and further future data, clinical trials may be justified.
\end{abstract}

Keywords: stem cell, brain tumor, glioma, tropism, growth suppression

\section{Introduction}

Glioma is the most common type of human primary brain tumor. Malignant gliomas constitute $22 \%-27 \%$ of all brain tumors. ${ }^{1}$ In spite of many technological advances in neurosurgery, neuroimaging, radiation therapy, and chemotherapy, the prognosis for patients with malignant gliomas is poor. The highly infiltrative nature of glioma cells is the major cause of their dismal prognosis. The glioma cells migrate from the core mass and produce secondary, microsatellite tumors in normal brain parenchyma. ${ }^{2}$

Removal of microsatellite tumors by surgery is not feasible, and these tumors are the seeds for recurrent tumor growth. ${ }^{3}$ Invasiveness is regulated by the interplay between secreted proteases (eg, cathepsins) and their endogenous inhibitors (cystatins). Cystatin E/M is a potent inhibitor of cathepsin B, which is frequently overexpressed in gliomas. ${ }^{4}$

One of the therapeutic strategies to treat glioma is the eradication of invading glioma cell microsatellite tumors before they develop into recurrent tumors. ${ }^{5}$ Some reports have shown that neural stem cell (NSC) transplantation may be useful in treating several central nervous system (CNS) diseases or injuries. Several groups have used NSCs to treat tumors that affect the CNS. ${ }^{6-9}$

The presence of tumor signals may influence the behavior of NSCs by virtue of their inherent migratory and tumor-trophic properties. This ability represents a new and potentially powerful approach in the treatment of invasive tumors and has been used as a delivery vehicle for targeting and disseminating therapeutic gene products throughout tumor sites. NSCs, by infiltrating the tumor mass selectively and aggressively, may help to overcome major obstacles that are being faced by current gene therapy strategies. ${ }^{2,10,11}$
Correspondence: Hossein Eskandary Neuroscience Research Center and Afzal Research Institute (NGO), Kerman, Iran $\mathrm{Tel}+983417690 \mathrm{I}$

Fax +983412455535

Email eskandary52@gmail.com 
Brain tumor tropism of NSCs can be used to deliver therapeutic molecules, such as genes, proteins, peptides, or small chemical molecules; however, clinical use of NSCs is limited by both ethical and logistical problems, including their isolation and their immunological compatibility in allogenic transplantation. ${ }^{12,13}$ Therefore, it is essential to find new sources of easily accessible stem cells with tumortargeting properties which are also useful for autologous transplantation.

The factors involved in brain tumor tropism of NSCs, and the interactions of NSCs within the tumor environment, are not well known. ${ }^{2}$ From the perspective of viewing stem cell biology as a means to track and help in the eradication of tumors, we have reviewed the literature to highlight the information on the mechanism of organization, regulation, and function of stem cell tropism and tumor growth suppression in glioma brain tumors. At this point nearly all of the data are limited to in vitro and animal studies and there is no evidence of clinical trials.

\section{Stem cell administration, fate and distribution around gliomas}

It has been shown that stem cells exhibit extensive tropism toward tumor sites and infiltrate tumor foci when implanted intraventricularly and intracranially within normal tissue on the side of a lesion, into the contralateral hemisphere, and through the peripheral, intravascular circulation. ${ }^{14-16}$

Intravascular delivery of stem cells is advantageous because it obviates invasive surgical interventions and because repeated injections over an extended period are clinically feasible. In the study by Nakamizo et al, injection of IFN- $\beta$ from human mesenchymal stem cells into the internal carotid artery significantly increased survival of animals bearing established intracranial gliomas. ${ }^{15}$ Interestingly, the authors found that when human mesenchymal stem cells (hMSCs) were injected into tail veins, the majority of stem cells were filtered by the lungs. Brown et al have suggested that intravascular administration of NSCs was an effective delivery vehicle for targeting and disseminating therapeutic agents to invasive tumors of neural and nonneural origin, both within and outside the brain. ${ }^{17}$ Tang et al showed that most NSCs migrate to the brain tumor through an intraventricular path. ${ }^{16}$ Based on the study by Kim et al, 50 minutes after contralateral hemispheric NSC injection, around $10 \%$ of the NSCs migrated to the tumor region. Five days after injection, the number of NSCs increased slowly, reaching a significant increase by 15 days post-injection. Changes in tumor volume showed similar patterns. The rate of NSC migration was approximately $175 \mu \mathrm{m} / \mathrm{min}$. In the absence of in vivo tumor cell inoculation, the number of NSCs increased approximately 1.7 -fold during day one; however, the proliferation of NSCs began to decrease 5 days after injection. ${ }^{18}$

Nakamizo et al found that the intratumoral injection of $2.5 \times 10^{4} \mathrm{hMSCs}$ did not extend animal survival. Based on this, they concluded that at least $25 \%$ of cells $\left(2.5 \times 10^{5}\right.$ hMSCs interferon [IFN]- $\beta$ ) must have integrated into the tumor for a significant increase in animal survival, so intraarterial injection of at least $1 \times 10^{6} \mathrm{hMSCs}$ is needed. ${ }^{15}$ Although the beneficial effects of different stem cells have been shown in animal models to some extent, the most effective type of stem cell, the more convenient and efficient route of delivery and the optimal number of stem cells in treatment of gliomas have not yet been elucidated and require further research.

\section{Mechanism of stem cell migration toward gliomas}

Although the brain is completely formed and integrated a few weeks after birth, it maintains some degree of plasticity throughout life, including axonal remodeling, synaptogenesis, neural cell birth, migration, and integration. The dentate gyrus (DG) of the hippocampus and the subventricular zone (SVZ) are the two main neurogenic niches in the adult brain. Neural stem cells live in these structures and produce progenitors that migrate toward their ultimate locations, including the granular cell layer of the DG and olfactory bulb, respectively. ${ }^{19}$ However, the vast majority of adult glial progenitors reside outside the neurogenic niches (DG and SVZ). Under normal circumstances, these resident adult progenitors and their glial progeny do not migrate, although they can be stimulated to migrate under pathological conditions. ${ }^{19-21}$

The role of NSCs in both the physiological and pathological processes in the brain has not been clearly explained. Normal NSCs possess the capability to migrate extensively toward the tumor mass and to linger in and around neoplastic regions of the brain. ${ }^{10}$ The tropism of NSCs toward brain tumors may provide an additional tool for the treatment of malignant brain tumors. The creation of potential NSCbased therapies has been studied, and this type of therapy involves the delivery of gene products to specific areas of the CNS that can selectively target malignant brain tumor cells and maximize the capability of their delivery. ${ }^{12,14}$

Many brain tumor behaviors unexpectedly resemble the intrinsic properties of neural stem/progenitor cells..$^{22,23}$ 
This has generated recent concern about providing stem cells to help eliminate tumors. There is also concern about the fact that stem cell biology may be somehow integral to the origination and/or production of the neoplasm itself. Yet, based on the unrivalled efficiency of NSCs to migrate throughout the brain and target invasive tumors, the transplantation of NSCs offers a new potential therapeutic approach as a cell-based delivery system for gene therapy in brain tumors. On the one hand, both stem cells and cancer cells are thought to be capable of unlimited proliferation. Yet, on the other hand, many tumors and cancer cell lines express stem cell markers, suggesting that either cancer cells look like stem cells or those cancers contain stem-like cells. ${ }^{22,24,25}$

During development or after xenograft inoculation, normal NSCs also display high levels of motility throughout the brain. ${ }^{26,27}$ This property is especially discernible when NSCs are inoculated immediately after injury. ${ }^{8,28}$ or during tumorigenesis. ${ }^{9,29}$ Such findings provide evidence that NSCs and brain tumor stem cells may respond to migratory signals in similar ways. ${ }^{22}$

Cell migration is an important multistep process that leads to organism development, tissue repair, and regeneration. In addition, it drives disease progression in cancer and inflammation. ${ }^{30}$ Every step of the cell migration process relates to extracellular factors that act on the cell itself through molecular pathways and intracellular signaling cascades. $^{2}$

In normal brains, secreted proteins, which act as chemoattractants or chemorepellants, coupled with proteins that are implicated in cell-cell or cell-matrix interactions, play pivotal roles in the regulation of neural progenitor cell migration. In addition, recent data suggest that gliomas originate from the transformation of neural stem cells or progenitor cells, ${ }^{24,31-33}$ and that glioma cell infiltration reiterates key aspects of glial progenitor migration. The factors that are implicated in such cell migrations and recruitments are just beginning to be understood. Many observations show that brain lesions and neurological diseases provoke neural stem/progenitor cell migration toward altered structures, such as tumors. Inflammation, which has long been contemplated as thoroughly devastating to brain repair, is now known to produce some positive effects on stem/progenitor cell recruitment through the regulation of growth factor signaling and the secretion of a number of chemoattractant cytokines. This knowledge is critical for the development of new therapeutic strategies. ${ }^{19}$

Cytokines, such as vascular endothelial cell growth factors (VEGFs), tumor growth factors (TGFs), epidermal growth factors (EGFs), platelet-derived growth factors (PDGF), monocyte chemoattractant protein-1 (MCP-1), and interleukin-8 (IL-8), which are released from the neoplasm or inflammatory tissues, are all possible candidates for tropism of stem cells. ${ }^{34-36}$ Mesenchymal cells augment VEGF-induced angiogenesis in vitro. ${ }^{37}$ It is recognized that the factors released from cancer cells promote the movement of endothelial cell progenitors and stromal cell progenitors from the bone marrow toward the tumor ${ }^{38,39}$ or tissues surrounding the tumor, which enhances the formation of tumor-stroma. ${ }^{40}$

Gondi et al have observed that human umbilical cord blood stem cells (hUCBs) show tropism toward glioma cells in vitro, in vivo, and ex vivo. They concluded that this migration relies partially on the expression levels of plateletderived growth factor-D (PDGF-D) from glioma cells. These investigators have also pointed out that a local concentration gradient of PDGF-D is sufficient to cause migration of hUCBs toward brain slice cultures. ${ }^{41}$

\section{NSC tumor tropism}

NSCs show extensive tropism toward the tumor itself or toward the CNS degeneration. In vitro studies have shown that NSCs did not migrate toward sites where a needle was inserted to imitate tissue damage that takes place during the establishment of a tumor bed, but where the actual implantation of glioblastoma cells did not take place. ${ }^{10}$ This suggests that the tumor itself possesses at least some of the tropic cues necessary to cause NSC migration. Nonetheless, in other previously reported experimental situations in which significant neuronal death took place. ${ }^{42}$ NSC differentiation was altered by apparent trophic influences. Therefore, the signals to which the NSCs respond are most likely complex, from multiple sources, and may represent a mixture of attractants, adhesion molecules, substrate molecules and chemokines of broader biological significance. These findings suggest that migration can be unexpectedly extensive, even in an adult brain, and along non-stereotypical routes, if pathology (ie, a tumor) is present. ${ }^{10}$ Little is known about the signals and factors that influence the tumor tropism of NSCs or their interactions within the tumor environment. It has been speculated that soluble factors, which are over-expressed by tumor cells, may be an important signal for the long-range attraction of NSCs from distant sites.

Some empirical evidence indicates that upregulation of stromal cell-derived factor $(\mathrm{SDF}-1)^{43}$ and $\mathrm{VEGF}^{35}$ serve as soluble chemotactic factors that induce NSC tropism toward gliomas; however, the observation that even microsatellites 
and infiltrating glioma cells that are distant from the main tumor mass are targeted by NSCs suggests that additional local signals exist that guide NSCs. The migration of glioma cells during invasion is associated with a complex and continuous remodeling of the pre-existing normal extracellular matrix (ECM) of the brain. ${ }^{44}$ In vitro and in vivo studies have shown that unlike the pre-existing normal ECM, the ECM of gliomas and their migration pathways consist mainly of tenascin, fibronectin, laminin, vitronectin, and different types of collagen. ${ }^{44-47}$

Laminin, fibronectin, tenascin- $\mathrm{C}$, and collagen I are localized within the basement membrane of existing and newly formed blood vessels. ${ }^{44}$ These basement membrane proteins, which comprise the reconstituted basement membrane Matri$\mathrm{gel}^{\mathrm{TM}}$ (BD Biosciences, Franklin Lakes, NJ), allowed the migration of HB1.F3-hNSCs in monolayer migration assays. ${ }^{2}$

The ECM is extensively modified when gliomas progress and invade the brain. Ziu et $\mathrm{al}^{2}$ analyzed the effects of tumor-ECM compounds from six glioblastoma cell lines on NSC motility. They found that NSC migration was highly dependent on tumor-produced ECM. Laminin and tenascin-C were the most permissive and the strongest inducers of human NSC migration, respectively. Different components of ECM produced by glioma cells positively affect the degree of NSC adhesion and migration. They also suggested that NSC migration is modulated by the ECM of malignant gliomas. These findings showed that the ECM plays a crucial role in NSC migration toward tumor cells, which reinforced the idea that cell migration is a complex process. This is further supported by the fact that the migratory rate of HB1. F3-hNSCs on normal Matrigel was significantly higher than on growth factor-depleted Matrigel. ${ }^{2}$

\section{HMSC tumor tropism}

Nakamizo et al concluded that the cell-specific capacity of hMSCs to localize in human gliomas seems to be an intrinsic property of this cell type. The results from this study indicate that migration of hMSCs toward glioma tumors may be mediated, at least in part, by growth factors and chemokines; however, this group showed that despite the presence of a wide range of growth factors within tumors, there is selectivity of hMSCs for specific factors. For example, whereas fibroblast growth factor (FGF) and VEGF had little effect on hMSC migration, PDGF, EGF, and SDF-1a enhanced hMSC tropism. Moreover, a cocktail of antibodies that block PDGF-BB, EGF, and SDF-1a was able to attenuate the migration of hMSCs toward conditioned medium derived from U87 cells. ${ }^{15}$
Nakamura et al showed that cultured rat glioma cells stimulate the migration of rat MSCs. ${ }^{48}$ Soluble factors released from $9 \mathrm{~L}$ glioma cells mediated the activation of MSC migration. Cytokines, such as hepatocyte growth factor (HGF), ${ }^{36} \mathrm{VEGF}^{37,49} \mathrm{TGFs},{ }^{36,37,49,50} \mathrm{FGFs}^{36}{ }^{36} \mathrm{PGF},{ }^{36,51}$ MCP-1, and IL-8, ${ }^{52,53}$ which are released from the neoplasm or inflammatory tissues, are all possible candidates. These factors, which are secreted from cancer cells, encourage the migration of endothelial cell progenitors and stromal cell progenitors from the bone marrow toward the cancer bed or tissue surrounding the tumor and enhance the formation of tumor-stroma. Similar mechanisms would be expected for the migration of implanted MSCs and tumor-stromal formation in gliomas. Rat MSCs introduced into tumors were basically distributed at the border zones between normal rat brain parenchyma and tumors. ${ }^{48}$

In vitro Matrigel invasion assays showed that conditioned media from gliomas, but not from fibroblasts or astrocytes, supported the migration of hMSCs, and that PDGF, EGF, or SDF-1a, but not basic FGF or VEGF, enhanced hMSC migration. ${ }^{15}$

In order to evaluate the capability of hMSCs to track human gliomas, Nakamizo et al injected hMSCs directly into the opposite side of the cerebral hemisphere of an established human glioma and showed that the hMSCs were capable of migrating into the xenograft. ${ }^{15}$ hMSCs may integrate into glioma tumors to contribute to the mesenchymal elements of the tumor and differentiate into glial and neuronal cells. ${ }^{54,55}$ Despite the fact that hMSCs may provide a microenvironment that is favorable for tumor growth, they may also have the capacity as a cellular vehicle for delivery of therapeutic agents to glioma tumors. Further explanation of the fundamental mechanism of hMSCs tropism toward gliomas may give insights into methods that can be used to increase the efficiency of the engraftment process. ${ }^{15}$

\section{Modified stem cell therapy}

By using genetically modified stem cells to secrete antineoplastic compounds, it may be possible to achieve high levels of one or more chemotherapeutic agents at the site of a tumor.

Different approaches are being undertaken by several researchers, all of whom are focusing on a variety of potentially therapeutic genes that could exert better efficacy with fewer side effects when expressed in close proximity to the tumor mass. Antineoplastic compounds have been divided into three categories, including the 
prodrug-converting enzymes, viral vectors, and immuneresponse modulators. ${ }^{14}$

Genetic strategies are also being developed to deliver genetically engineered NSCs to the sites of brain tumors. Candidate genes include those that encode proteins that induce differentiation of neoplastic cells and/or their signal transduction mediators, cell cycle modulators, apoptosis-promoting agents, antiangiogenesis factors, immune-enhancing agents, and oncolytic factors. ${ }^{10,56}$

Lee et al evaluated the therapeutic efficacy of genetically modified NSCs encoding cytosine deaminase (CD) and IFN- $\beta$, a proinflammatory cytokine gene, in treating brain stem gliomas. They added 5-fluorocytosine to kill dividing cells, including human NSCs encoding CD and IFN- $\beta$. Histological analyses showed a 59\% reduction in tumor volume in the treated group, and apoptosis was 2.33 -fold higher in the treated group than in the control group. ${ }^{57}$ The therapeutic actions of CD and IFN- $\beta$ are different. $C D$ acts as prodrug-activating enzyme and IFN- $\beta$ has the antiangiogenic effect and immune response.$^{58}$ Nearly the same gene therapy with human NSCs had significant therapeutic benefit in experimental gliomas. ${ }^{59}$ In another study, NSCs were modified to produce CD. Administration of these NSCs caused an $80 \%$ reduction in tumor masses when animals were treated with the systemic prodrug 5 -fluorocytosine. ${ }^{17}$

Antitumor effects of intracranial administration of gene modified NSC expressing IL-4, ${ }^{12}$ IL-12, ${ }^{7}$ or tumor necrosis factor-related apoptosis-inducing ligand (TRAIL) ${ }^{6}$ have also been reported.

Gene modification of MSCs by infection with an adenoviral vector encoding human IL-2 clearly augmented the antitumor effect and further prolonged the survival of tumor-bearing rats. ${ }^{48}$

In experimental glioma models, Nakamizo et al found that hMSCs that were engineered to produce IFN- $\beta$ would provide a high degree of local intratumoral delivery, with a limited degree of systemic toxicity. They used an adenoviral vector to transfer the IFN- $\beta$ gene into hMSCs and found that these engineered hMSCs (hMSC IFN- $\beta$ ) released high levels of IFN- $\beta$ and were capable of directly killing human glioma cell lines that were grown in vitro. These studies provided the proof of principle that hMSCs can be engineered to release a soluble factor into brain tumors. The authors suggested that IFN- $\beta$ is itself a good therapeutic agent worthy of assessment in patients with gliomas. The same approach can be exploited in the delivery of other agents with antitumor activity. Methods to maximize transfection of therapeutic genes to
hMSCs and to separate transfected from nontransfected cells are challenges for the ultimate application of this and other stem cell approaches to treating tumors. ${ }^{15}$ Although their studies have focused on bone marrow-derived hMSCs, Lee et al have suggested that other cells in the bone marrow may also be useful as delivery vehicles for brain tumors. ${ }^{29}$

Using live imaging and tumor measurements, Goren et al reported that encapsulated hMSC-PEX (Pexin) injected next to glioblastoma tumors in nude mice significantly reduced tumor volume $(87 \%)$ and weight (83\%). The authors clearly demonstrated that hMSCs are the best cell type for microencapsulation cell-based therapy, which brought this technology closer to clinical application. ${ }^{60}$

Germano et al showed that modified embryonic stem cells expressing transgenic hTRAIL induced apoptosis in human malignant glioma cells while sparing normal cells. ${ }^{61}$

All of the above observations imply that modified stem cells carrying therapeutic genes may successfully suppress the growth of glioma tumors.

\section{Unmodified stem cell therapy}

NSCs have been shown to mobilize and juxtapose themselves along aggressively advancing tumor cells. ${ }^{10}$ Glass et al demonstrated that endogenous neural precursors migrated from the SVZ toward a tumor mass and surrounded the bulk of the tumor in an established intracranial glioma mouse model. Furthermore, endogenous neural precursor cells did not manifest a pathotropic movement when implanted with other non-neoplastic lesions, which suggests a specific tropism toward brain tumors. Unmodified NSCs that were cocultured with glioblastoma multiform cells showed both a suppression of tumor growth and an induction of tumor cell apoptosis in mice, which improved their survival. ${ }^{62}$ Another previous study has provided evidence of the antitumor activity of NSCs and showed their migration toward tumor cells. ${ }^{63}$

Staflin et al showed that transplanted neural progenitor cells respond to queues from a tumor, home to, and exert an antitumor effect on the pre-established glioma. Transplanted NSCs significantly decreased the tumor volume by approximately $67 \%$ compared to untreated, control tumors after 1 to 2 weeks. Furthermore, these early effects could be translated into increased survival times of the animals treated with neural progenitor cell grafts 3 days after intrastriatal tumor injection. In contrast, there was no activation or migration of endogenous SVZ neuroblasts in response to intrastriatal syngeneic tumors. They concluded 
that neuronal precursor cells possess the ability to influence tumor growth and respond to queues from the tumor or tumor microenvironment, thereby demonstrating cross-talk between the cells. ${ }^{64}$

To evaluate whether the increased survival that was observed after inoculation of MSC-IL2 or MSC was associated with the inhibition of tumor growth, Nakamura et al monitored tumor growth volume by magnetic resonance imaging (MRI) every 7 days after intracranial injection of tumors. The $9 \mathrm{~L}$ glioma was clearly visible as an enhanced area in the coronal section of the brain. Fourteen days after tumor inoculation, glioma growth progression was observed in the brain of untreated rats and reached a lethal volume. In contrast, significantly smaller tumor volumes were present in brains of animals treated with MSC-IL2 or unmodified MSCs $(P<0.01$, compared with untreated controls 14 days after tumor inoculation). A significant difference in tumor volume was not observed between the groups treated with unmodified MSCs and MSCs-IL2s by day 14; however, the therapeutic effect of MSCs expressing IL-2 was clearly visible by MRI 21 days after tumor injection. At this time, tumors treated with unmodified MSCs had reached near-lethal volumes, but those tumors treated with IL-2-expressing MSCs resulted in smaller tumors. The observed changes in glioma tumor volume were consistent with the survival durations in the different treatment groups. The prolonged survival in glioma-bearing rats treated in this way might depend on a direct antitumor effect of the MSCs themselves. $^{48}$

It has been reported that in the cerebral infarction model, implanted MSCs mediate neural protection through the inhibition of neuronal apoptosis, and this protective effect is thought to be due to neurotrophic factors, such as nerve growth factor (NGF), which are released from MSCs. ${ }^{65}$ The protective effect of MSCs on normal brain parenchyma may also contribute to prolonged survival of glioma-bearing rats treated by MSC implantation. ${ }^{66}$

MSCs generate several neurotrophic factors, including NGF, which can induce differentiation and growth inhibition of rat glioma cells in vitro. ${ }^{48,67}$ Kang et al demonstrated another cytotoxic mechanism of rat MSCs, which involved the differentiation of rat MSCs into immune effector cells; ${ }^{68}$ however, differentiation of hUCB-derived MSCs into immune effector cells has not been demonstrated, although a variety of cytokines could activate these cells. ${ }^{69}$

MSCs have been found to secrete large amounts of angiogenic factors, such as angiopoietin-1 (Ang1). Ang1 inhibits tumor-vascular leakage and tumor growth in vivo. ${ }^{70}$ Ang1 released from MSCs influences the antitumor effects of MSCs. Ang1 may also protect brain parenchyma from lethal cerebral edema via suppression of vascular leakage. MSCs mainly localize between the edge of the tumor and normal parenchyma and make a capsule-like structure. This capsule-like structure of MSCs may act as a barrier that prevents the spread of glioma cells into normal parenchyma. Thus, implantation of MSCs may be beneficial for the treatment of gliomas both because of their antitumor effects and their protective effects on normal brain tissue. ${ }^{66}$

Pisati et al evaluated tumor targeting and anti-tumor activity of human skin-derived stem cells (hSDSCs). This group showed that when hSDSCs were injected directly into glioblastomas in mice, the hSDSCs distributed themselves throughout the tumor mass and reduced tumor vessel density and angiogenic sprouts. The hSDSCs also differentiated into pericyte cells and produced high levels of human actor- $\beta 1$ with low expression of VEGF, all of which may decrease tumor growth and prolong animal survival. $^{67}$

The ability of hUCBs to inhibit established intracranial tumors was observed by Gondi et al The results of this study demonstrated that hUCBs are capable of inducing apoptosis in human glioma cells. ${ }^{34,41}$

\section{Endogenous stem cells}

Many observations have shown that brain lesions and neurological diseases trigger neural stem/progenitor cell activation and migration toward the pathological structures. Normal NSCs exhibit a high degree of motility throughout the brain after xenograft injection. ${ }^{26,27}$ In fact, the results of one study indicate that endogenous precursor cells are attracted by tumor cells, the presence of precursor cells is anti-tumorigenic, and this cellular interaction decreases with age. ${ }^{62}$

The factors implicated in such cell migrations and recruitments are just beginning to be understood. Some factors have been reported to enhance stem cell migration toward glioblastoma cells, such as transmembrane protein 18 , MCP-1, MIP-1, and IL-8. ${ }^{50,71}$ These factors and ischemic cerebral tissue enhance human bone marrow stromal cell migration in interface culture..$^{50,53}$

A recombinant human TGF- $\beta 1$ fusion protein with a collagen-binding domain promotes migration, growth, and differentiation of bone marrow mesenchymal cells. ${ }^{50}$ 
It has long been considered that inflammation is largely devastating for brain repair, and it is now known to produce some positive effects on stem/progenitor cell recruitment via the regulation of growth factor signaling and the secretion of a number of chemoattractant cytokines. This knowledge is crucial for the development of new therapeutic strategies. One of these strategies could consist of increasing the mobilization of endogenous progenitor cells that could replace lost cells and improve functional recovery. It is not yet known why newly added neurons do not originate directly in the place in which they need to be located. Progenitor cell migration may provide an additional level of control for cell positioning, and the preservation of stem cell niches also represents a potential source of cells for brain repair. Yet, this may be costly for the organism, and it also requires specific features that restrict the structures to locations where they can persist. This idea implies that cells need to be able to migrate from these discrete niches to their final destinations. ${ }^{19}$

\section{Concluding remarks}

\section{Summary}

In this review, we concentrated on stem cell migration toward glioma tumors and provided some suggested mechanisms by which these stem cells may suppress tumor growth. Brain lesions, tumors, and neurological diseases trigger migration of neural stem cell/progenitor cells toward these altered structures. An understanding of the factors involved in to such cell migration events is a necessary step in delineating the critical pathways that control NSC tropism. The factors that are involved in migration of NSCs can be divided into two main groups: chemoattractant cytokines, including HGF, EGF, VEGF, TGF, FGF, PDGF, MPC-1, IL-8, IL-4, and SDF-1 $\mathrm{a}^{15,35-}$ $37,41,43,49,50,72$ and extracellular matrix compounds, such as tenascin, fibronectin, laminin, vitronectin, various types of collagen, and hyaluronic acid (Figure 1). ${ }^{2,44-47,73,74}$

It seems that there is selectivity of hMSCs for specific factors. For example, FGF and VEGF have little effect on hMSC migration, but PDGF, EGF, and SDF-1a enhance hMSC tropism. ${ }^{15}$

While the mechanisms by which modified stem cells suppress tumor growth are more often studied (see Achanta et $\mathrm{al}^{14}$ ), the precise mechanisms by which unmodified stem cells suppress tumor growth are not completely understood. We suppose that biochemical immunological and physical effects are responsible for growth inhibition of glioma tumor.

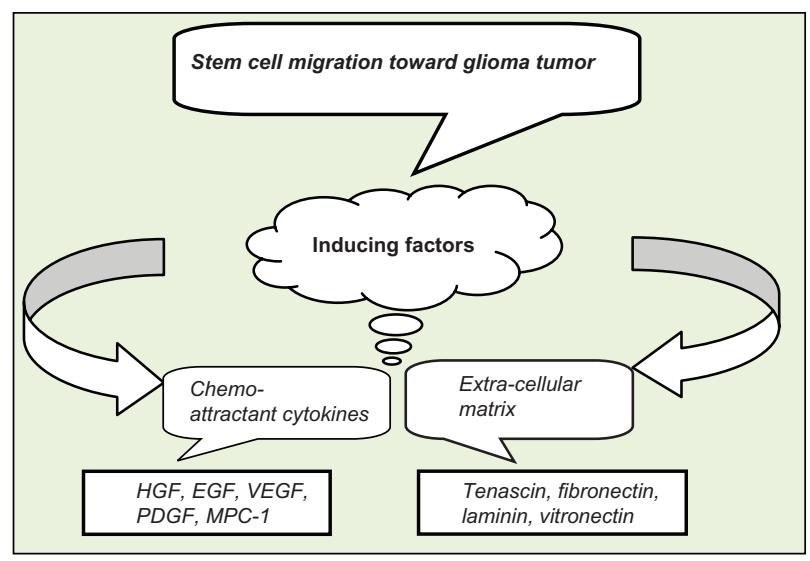

Figure I Stem cell migration is related to two groups of inducing factors of chemoattractant cytokines and extracellular matrix.

A few mechanisms are suggested in some reports, including the following (Figure 2):

1. Differentiation and growth inhibition of glioma cells in vitro, which may be due to the production of NGF by the MSCs. ${ }^{48,65,75}$

2. A cytotoxic mechanism that involves the differentiation of rat MSCs into immune effector cells. ${ }^{68,69}$

3. Apoptosis, in which hUCBs may be capable of inducing apoptosis in human glioma cells..$^{34,41,76}$

4. Inhibition of tumor growth and vascular leakage, which may be caused by the production of Ang $1 .{ }^{70}$ Ang 1 may also protect the brain parenchyma from lethal cerebral edema by reducing vascular leakage. ${ }^{48,70}$ Ang 1 inhibits tumor-vascular leakage and tumor growth in vivo. ${ }^{70}$

5. Formation of a barrier in which MSCs may prevent the spread of glioma cells into normal parenchyma. ${ }^{48}$

\section{Future insights}

An increasing knowledge about the factors that are necessary for stem cell survival, attraction, and their interactions with the tumor environment is necessary for the development of rational therapeutic strategies using stem cell technology. ${ }^{2}$ Some of the following issues should be considered in further studies of cell-based therapies for the treatment of tumors:

1. Stem cell type: we are currently performing the experiments necessary to determine whether there is any difference in the potency of tumor growth suppression and tropism among multiple types of stem cells.

2. Factors that induce stem cell migration: different factors may have varying effects on the tropism of different types of stem cells. 


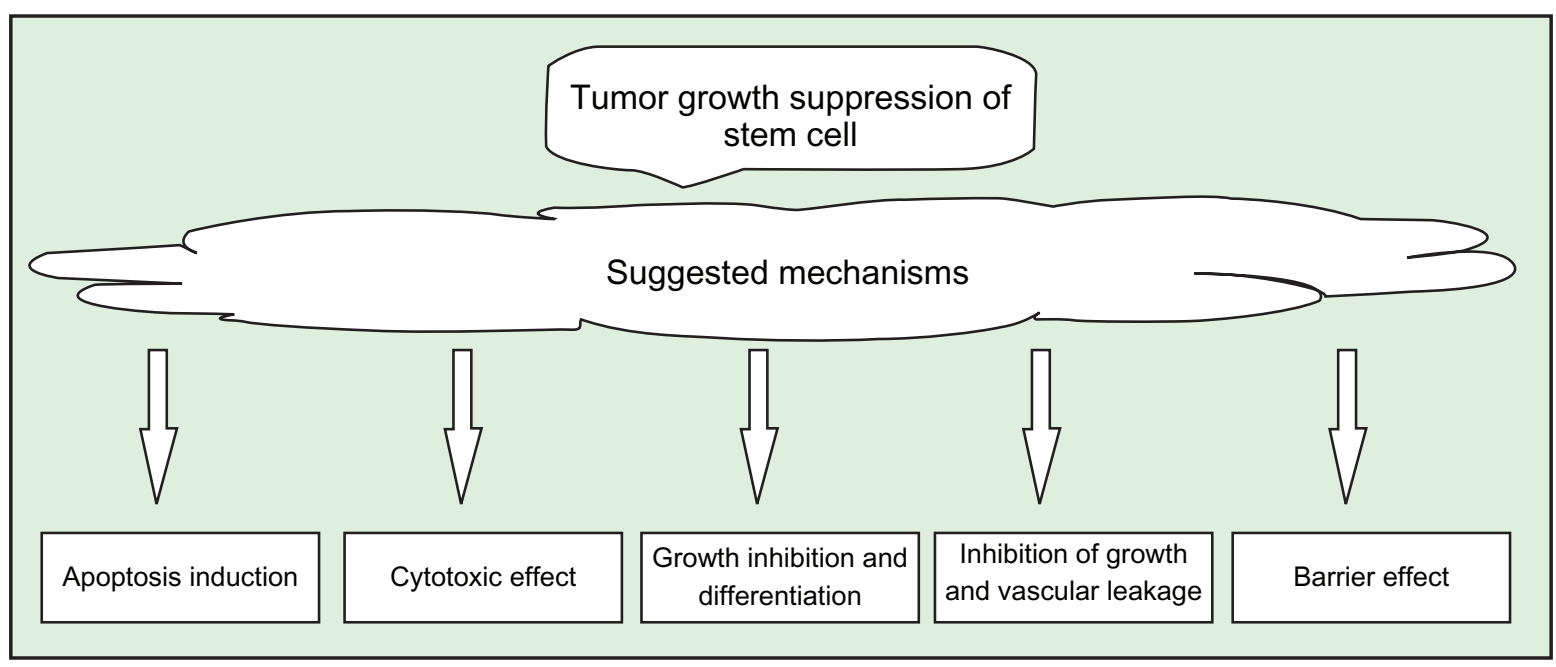

Figure 2 Some suggested mechanisms of tumor growth suppression of unmodified stem cells.

3. Timing of the effect: there was no significant difference in tumor volume between groups treated with unmodified MSCs and IL-2-expressing MSCs after 14 days of treatment. The therapeutic effect of the IL-2 gene modification was, however, clearly visible by MRI 21 days after tumor inoculation. ${ }^{15}$

4. Route of administration: importantly, regional delivery of IFN- $\beta$-expressing hMSCs by injection into the internal carotid artery significantly extended the survival of animals harboring established intracranial gliomas. Conversely, intravenous injection of IFN- $\beta$ did not extend animal survival. ${ }^{15}$

5. Dose dependency of antitumor effects: the most appropriate dose of stem cells for treatment of tumors remains unknown and needs to be studied. Nakamizo et al estimate that at least $25 \%$ of the cells $\left(2.5 \times 10^{5}\right)$ must have integrated into the tumor for a significant effect to be observed on tumor growth suppression. ${ }^{15}$

6. Endogenous production of stem cells: considering that normal stem cells exhibit a high degree of motility throughout the brain, and that tumor cells attract endogenous precursor cells, this innate behavior of endogenous NSCs may be helpful for suppressing tumor growth. If this is true, enhancing the endogenous production of stem cells may be a considerable issue for further studies.

7. Neutralization of the growth-inhibitory components of endogenous neuronal stem cells: this may help to increase the capacity of attraction.

8. Microenvironmental changes: chemical, ${ }^{77}$ physical, and mechanical interactions can affect the ECM, and cell growth and differentiation can be normalized by modulating cell adhesion to the ECM. Embryonic tissues may reverse cancerous growth by restoring these normal microenvironmental cues, ${ }^{78}$ and it is possible that the migration of either endogenous or exogenous stem cells toward gliomas may change the microenvironment and suppress tumor growth.

\section{Acknowledgments}

The authors gratefully acknowledge Mrs Jamileh Mahdavi, Dr Seyed Pouyan Jalali and Dr Mohammad Eskandari for their editorial assistance.

\section{Disclosure}

The authors report no conflicts of interest in this work.

\section{References}

1. Surawicz TS, McCarthy BJ, Kupelian V, Jukich PJ, Bruner JM, Davis FG. Descriptive epidemiology of primary brain and CNS tumors: results from the Central Brain Tumor Registry of the United States, 1990-1994. Neuro Oncol. 1999;1(1):14-25.

2. Ziu M, Schmidt NO, Cargioli TG, Aboody KS, Black PM, Carroll RS. Glioma-produced extracellular matrix influences brain tumor tropism of human neural stem cells. J Neurooncol. 2006;79(2):125-133.

3. Giese A, Bjerkvig R, Berens ME, Westphal M. Cost of migration: invasion of malignant gliomas and implications for treatment. J Clin Oncol. 15 2003;21(8):1624-1636.

4. Qiu J, Ai L, Ramachandran C, et al. Invasion suppressor cystatin E/M (CST6): high-level cell type-specific expression in normal brain and epigenetic silencing in gliomas. Lab Invest. 2008;88(9):910-925.

5. Dunn IF, Black PM. The neurosurgeon as local oncologist: cellular and molecular neurosurgery in malignant glioma therapy. Neurosurgery. 2003;52(6):1411-1422; discussion 1422-1414.

6. Ehtesham M, Kabos P, Gutierrez MA, et al. Induction of glioblastoma apoptosis using neural stem cell-mediated delivery of tumor necrosis factor-related apoptosis-inducing ligand. Cancer Res. 2002;62(24): 7170-7174.

7. Ehtesham M, Kabos P, Kabosova A, Neuman T, Black KL, Yu JS. The use of interleukin 12-secreting neural stem cells for the treatment of intracranial glioma. Cancer Res. 2002;62(20):5657-5663. 
8. Hayashi T, Iwai M, Ikeda T, et al. Neural precursor cells division and migration in neonatal rat brain after ischemic/hypoxic injury. Brain Res. 2005;1038(1):41-49.

9. Li S, Gao Y, Tokuyama T, et al. Genetically engineered neural stem cells migrate and suppress glioma cell growth at distant intracranial sites. Cancer Lett. 2007;251(2):220-227.

10. Aboody KS, Brown A, Rainov NG, et al. Neural stem cells display extensive tropism for pathology in adult brain: evidence from intracranial gliomas. Proc Natl Acad Sci U S A. 2000;97(23):12846-12851.

11. Kim SU. Genetically engineered human neural stem cells for brain repair in neurological diseases. Brain Dev. 2007;29(4):193-201.

12. Benedetti S, Pirola B, Pollo B, et al. Gene therapy of experimental brain tumors using neural progenitor cells. Nat Med. 2000;6(4): 447-450.

13. Bosman FT, Stamenkovic I. Functional structure and composition of the extracellular matrix. J Pathol. 2003;200(4):423-428.

14. Achanta P, Sedora Roman NI, Quinones-Hinojosa A. Gliomagenesis and the use of neural stem cells in brain tumor treatment. Anticancer Agents Med Chem. 2010;10(2):121-130.

15. Nakamizo A, Marini F, Amano T, et al. Human bone marrow-derived mesenchymal stem cells in the treatment of gliomas. Cancer Res. 2005; 65(8):3307-3318.

16. Tang Y, Shah K, Messerli SM, Snyder E, Breakefield X, Weissleder R. In vivo tracking of neural progenitor cell migration to glioblastomas. Hum Gene Ther. 2003;14(13):1247-1254.

17. Brown AB, Yang W, Schmidt NO, et al. Intravascular delivery of neural stem cell lines to target intracranial and extracranial tumors of neural and non-neural origin. Hum Gene Ther. 2003;14(18): 1777-1785.

18. Kim JH, Lee JE, Kim SU, Cho KG. Stereological analysis on migration of human neural stem cells in the brain of rats bearing glioma. Neurosurgery. 2010;66(2):333-342; discussion 342.

19. Cayre M, Canoll P, Goldman JE. Cell migration in the normal and pathological postnatal mammalian brain. Prog Neurobiol. 2009;88(1): 41-63.

20. Goh E, Ma D, Ming G, Song H. Adult neural stem cells and repair of the adult central nervous system. J Hematother Stem Cell Res. 2003; 12(6):671-679.

21. Ma D, Bonaguidi M, Ming G, Song H. Adult neural stem cells in the mammalian central nervous system. Cell research. 2009;19(6): 672-682.

22. Colleoni F, Torrente Y. The new challenge of stem cell: brain tumour therapy. Cancer Lett. 2008;272(1):1-11.

23. Vescovi AL, Galli R, Reynolds BA. Brain tumour stem cells. Nat Rev Cancer. 2006;6(6):425-436.

24. Fomchenko EI, Holland EC. Stem cells and brain cancer. Exp Cell Res. 2005;306(2):323-329.

25. Galderisi U, Cipollaro M, Giordano A. Stem cells and brain cancer. Cell Death Differ. 2006;13(1):5-11.

26. Dirks PB. Glioma migration: clues from the biology of neural progenitor cells and embryonic CNS cell migration. J Neurooncol. 2001;53(2):203-212.

27. Watson DJ, Walton RM, Magnitsky SG, Bulte JW, Poptani H, Wolfe JH. Structure-specific patterns of neural stem cell engraftment after transplantation in the adult mouse brain. Hum Gene Ther. 2006; 17(7):693-704.

28. Chang YC, Shyu WC, Lin SZ, Li H. Regenerative therapy for stroke. Cell Transplant. 2007;16(2):171-181.

29. Lee J, Elkahloun AG, Messina SA, et al. Cellular and genetic characterization of human adult bone marrow-derived neural stem-like cells: a potential antiglioma cellular vector. Cancer Res. 2003;63(24): 8877-8889.

30. Ridley AJ, Schwartz MA, Burridge K, et al. Cell migration: integrating signals from front to back. Science. 2003;302(5651): 1704-1709.

31. Panagiotakos G, Tabar V. Brain tumor stem cells. Curr Neurol Neurosci Rep. 2007;7(3):215-220.
32. Shiras A, Chettiar ST, Shepal V, Rajendran G, Prasad GR, Shastry P. Spontaneous transformation of human adult nontumorigenic stem cells to cancer stem cells is driven by genomic instability in a human model of glioblastoma. Stem Cells. 2007;25(6):1478-1489.

33. Xie Z. Brain tumor stem cells. Neurochem Res. 2009;34(12): 2055-2066.

34. Gondi CS, Gogineni VR, Chetty C, et al. Induction of apoptosis in glioma cells requires cell-to-cell contact with human umbilical cord blood stem cells. Int J Oncol. 2010;36(5):1165-1173.

35. Schmidt N, Przylecki W, Yang W, et al. Brain tumor tropism of transplanted human neural stem cells is induced by vascular endothelial growth factor. Neoplasia (New York, NY). 2005;7(6):623.

36. Yamamoto S, Wakimoto H, Aoyagi M, Hirakawa K, Hamada H. Modulation of motility and proliferation of glioma cells by hepatocyte growth factor. Jpn J Cancer Res. 1997;88(6):564-577.

37. Tille JC, Pepper MS. Mesenchymal cells potentiate vascular endothelial growth factor-induced angiogenesis in vitro. Exp Cell Res. 2002;280(2): $179-191$.

38. Coussens LM, Werb Z. Inflammation and cancer. Nature. 2002;420 (6917):860-867.

39. De Palma M, Venneri MA, Roca C, Naldini L. Targeting exogenous genes to tumor angiogenesis by transplantation of genetically modified hematopoietic stem cells. Nat Med. 2003;9(6):789-795.

40. Weaver VM, Fischer AH, Peterson OW, Bissell MJ. The importance of the microenvironment in breast cancer progression: recapitulation of mammary tumorigenesis using a unique human mammary epithelial cell model and a three-dimensional culture assay. Biochem Cell Biol. 1996;74(6):833-851.

41. Gondi CS, Veeravalli KK, Gorantla B, et al. Human umbilical cord blood stem cells show PDGF-D-dependent glioma cell tropism in vitro and in vivo. Neuro Oncol. 2010;12(5):453-465.

42. Snyder E, Yoon C, Flax J, Macklis J. Multipotent neural precursors can differentiate toward replacement of neurons undergoing targeted apoptotic degeneration in adult mouse neocortex. Proc Natl Acad Sci US A. 1997;94(21):11663.

43. Ehtesham M, Yuan X, Kabos P, et al. Glioma tropic neural stem cells consist of astrocytic precursors and their migratory capacity is mediated by CXCR4. Neoplasia. 2004;6(3):287-293.

44. Giese A, Westphal M. Glioma invasion in the central nervous system. Neurosurgery. 1996;39(2):235-250; discussion 250-232.

45. Bellail AC, Hunter SB, Brat DJ, Tan C, Van Meir EG. Microregional extracellular matrix heterogeneity in brain modulates glioma cell invasion. Int J Biochem Cell Biol. 2004;36(6):1046-1069.

46. Friedlander DR, Zagzag D, Shiff B, et al. Migration of brain tumor cells on extracellular matrix proteins in vitro correlates with tumor type and grade and involves alphaV and beta1 integrins. Cancer Res. 1996; 56(8):1939-1947.

47. Mahesparan R, Read TA, Lund-Johansen M, Skaftnesmo KO, Bjerkvig R, Engebraaten O. Expression of extracellular matrix components in a highly infiltrative in vivo glioma model. Acta Neuropathol. 2003;105(1):49-57.

48. Nakamura K, Ito Y, Kawano Y, et al. Antitumor effect of genetically engineered mesenchymal stem cells in a rat glioma model. Gene Ther. 2004;11(14):1155-1164.

49. Schichor C, Birnbaum T, Etminan N, et al. Vascular endothelial growth factor A contributes to glioma-induced migration of human marrow stromal cells (hMSC). Exp Neurol. 2006;199(2): 301-310.

50. Andrades JA, Han B, Becerra J, Sorgente N, Hall FL, Nimni ME. A recombinant human TGF-betal fusion protein with collagen-binding domain promotes migration, growth, and differentiation of bone marrow mesenchymal cells. Exp Cell Res. 1999;250(2):485-498.

51. Yu J, Ustach C, Kim HR. Platelet-derived growth factor signaling and human cancer. J Biochem Mol Biol. 2003;36(1):49-59.

52. Wang L, Li Y, Chen J, et al. Ischemic cerebral tissue and MCP-1 enhance rat bone marrow stromal cell migration in interface culture. Exp Hematol. 2002;30(7):831-836. 
53. Wang L, LiY, Chen X, et al. MCP-1, MIP-1, IL-8 and ischemic cerebral tissue enhance human bone marrow stromal cell migration in interface culture. Hematology. 2002;7(2):113-117.

54. Kopen GC, Prockop DJ, Phinney DG. Marrow stromal cells migrate throughout forebrain and cerebellum, and they differentiate into astrocytes after injection into neonatal mouse brains. Proc Natl Acad Sci U S A. 1999;96(19):10711-10716.

55. Woodbury D, Schwarz EJ, Prockop DJ, Black IB. Adult rat and human bone marrow stromal cells differentiate into neurons. J Neurosci Res. 2000;61(4):364-370.

56. Yao K, Komata T, Kondo Y, Kanzawa T, Kondo S, Germano I. Molecular response of human glioblastoma multiforme cells to ionizing radiation: cell cycle arrest, modulation of cyclin-dependent kinase inhibitors, and autophagy. J Neurosurg. 2003;98(2):378-384.

57. Lee DH, Ahn Y, Kim SU, et al. Targeting rat brainstem glioma using human neural stem cells and human mesenchymal stem cells. Clin Cancer Res. 2009;15(15):4925-4934.

58. Kim SK, Kim SU, Park IH, et al. Human neural stem cells target experimental intracranial medulloblastoma and deliver a therapeutic gene leading to tumor regression. Clin Cancer Res. 2006;12(18): $5550-5556$.

59. Ito S, Natsume A, Shimato S, et al. Human neural stem cells transduced with IFN-beta and cytosine deaminase genes intensify bystander effect in experimental glioma. Cancer Gene Ther. 2010;17(5):299-306.

60. Goren A, Dahan N, Goren E, Baruch L, Machluf M. Encapsulated human mesenchymal stem cells: a unique hypoimmunogenic platform for long-term cellular therapy. Faseb J. 2010;24(1):22-31.

61. Germano IM, Uzzaman M, Benveniste RJ, Zaurova M, Keller G. Apoptosis in human glioblastoma cells produced using embryonic stem cell-derived astrocytes expressing tumor necrosis factor-related apoptosis-inducing ligand. J Neurosurg. 2006;105(1):88-95.

62. Glass R, Synowitz M, Kronenberg G, et al. Glioblastoma-induced attraction of endogenous neural precursor cells is associated with improved survival. J Neurosci. 2005;25(10):2637-2646.

63. Staflin K, Honeth G, Kalliomaki S, Kjellman C, Edvardsen K, Lindvall M. Neural progenitor cell lines inhibit rat tumor growth in vivo. Cancer Res. 2004;64(15):5347-5354.

64. Staflin K, Lindvall M, Zuchner T, Lundberg C. Instructive cross-talk between neural progenitor cells and gliomas. J Neurosci Res. 2007; 85(10):2147-2159.

65. Li Y, Chen J, Chen XG, et al. Human marrow stromal cell therapy for stroke in rat: neurotrophins and functional recovery. Neurology. 2002; 59(4):514-523.
66. Hamada H, Kobune M, Nakamura K, et al. Mesenchymal stem cells (MSC) as therapeutic cytoreagents for gene therapy. Cancer Sci. 2005; 96(3):149-156.

67. Pisati F, Belicchi M, Acerbi F, et al. Effect of human skin-derived stem cells on vessel architecture, tumor growth, and tumor invasion in brain tumor animal models. Cancer Res. 2007;67(7):3054-3063.

68. Kang SG, Jeun SS, Lim JY, et al. Cytotoxicity of rat marrow stromal cells against malignant glioma cells. Childs Nerv Syst. 2005;21(7): $528-538$.

69. Kang SG, Jeun SS, Lim JY, et al. Cytotoxicity of human umbilical cord blood-derived mesenchymal stem cells against human malignant glioma cells. Childs Nerv Syst. 2008;24(3):293-302.

70. Stoeltzing O, Ahmad SA, Liu W, et al. Angiopoietin-1 inhibits vascular permeability, angiogenesis, and growth of hepatic colon cancer tumors. Cancer Res. 2003;63(12):3370-3377.

71. Jurvansuu J, Zhao Y, Leung DS, et al. Transmembrane protein 18 enhances the tropism of neural stem cells for glioma cells. Cancer Res. 2008;68(12):4614-4622.

72. Rempel SA, Dudas S, Ge S, Gutierrez JA. Identification and localization of the cytokine SDF1 and its receptor, CXC chemokine receptor 4, to regions of necrosis and angiogenesis in human glioblastoma. Clin Cancer Res. 2000;6(1):102-111.

73. Ohnishi T, Hiraga S, Izumoto S, et al. Role of fibronectin-stimulated tumor cell migration in glioma invasion in vivo: clinical significance of fibronectin and fibronectin receptor expressed in human glioma tissues. Clin Exp Metastasis. 1998;16(8):729-741.

74. Sobeih MM, Corfas G. Extracellular factors that regulate neuronal migration in the central nervous system. Int J Dev Neurosci. 2002; 20(3-5):349-357.

75. Kimura S, Yoshino A, Katayama Y, Watanabe T, Fukushima T. Growth control of C6 glioma in vivo by nerve growth factor. $J$ Neurooncol. 2002;59(3):199-205.

76. Dasari VR, Velpula KK, Kaur K, et al. Cord blood stem cell-mediated induction of apoptosis in glioma downregulates X-linked inhibitor of apoptosis protein (XIAP). PLoS One. 2010;5(7):e11813.

77. Charles N, Ozawa T, Squatrito M, et al. Perivascular nitric oxide activates notch signaling and promotes stem-like character in PDGFinduced glioma cells. Cell Stem Cell. 2010;6(2):141-152.

78. Ingber D. Can cancer be reversed by engineering the tumor microenvironment? 2008.
Biologics: Targets \& Therapy

\section{Publish your work in this journal}

Biologics: Targets \& Therapy is an international, peer-reviewed journal focusing on the patho-physiological rationale for and clinical application of Biologic agents in the management of autoimmune diseases, cancers or other pathologies where a molecular target can be identified. This journal is indexed on PubMed Central, CAS, EMBase, Scopus

\section{Dovepress}

and the Elsevier Bibliographic databases. The manuscript management system is completely online and includes a very quick and fair peerreview system, which is all easy to use. Visit http://www.dovepress. com/testimonials.php to read real quotes from published authors. 